\title{
Andexanet Alfa and its Clinical Application
}

\author{
Fauve A Noordergraaf ${ }^{1}$ and Marco Alings ${ }^{1,2}$ \\ 1. Department of Cardiology, Amphia Hospital, Breda, Netherlands; 2. Heart \& Lung Division, Utrecht University Medical Center, \\ Utrecht, Netherlands
}

DOI: https://doi.org/10.17925/HI.2020.14.1.20

\begin{abstract}
$\mathrm{F}$ actor Xa (FXa) inhibitors are widely used for stroke prevention in patients with nonvalvular atrial fibrillation, and for the treatment and prevention of deep venous thrombosis and pulmonary embolism. Compared with warfarin, individual FXa inhibitors are associated with a lower risk of major bleeding. Nevertheless, bleeding remains a feared complication of any anticoagulant therapy. Despite their demonstrated safety, implementation of FXa inhibitors in clinical practice may have been limited by the lack of a specific antidote. Recently, however, the United States Food and Drug Administration and the European Medicines Agency approved andexanet alfa for reversal of anticoagulation in patients treated with rivaroxaban or apixaban who have life-threatening or uncontrolled bleeding. This review will discuss andexanet alfa's mode of action, indication for use and efficacy, with a focus on its appropriate use in clinical practice. Unnecessary usage should be prevented as this may compromise patient safety. Assessment of potentially suitable patients by a multidisciplinary team, use according to the institutional protocol and central storage, all contribute to proper use of andexanet alfa. A practical tool to direct appropriate use of andexanet alfa is proposed.
\end{abstract}

\section{Keywords}

Andexanet alfa, factor Xa inhibitors, major bleeding, reversal agent

Disclosures: Fauve A Noordergraaf has no financial or non-financial relationships or activities to declare in relation to this article. Marco Alings has received consulting fees from Portola Pharmaceuticals.

Review Process: Double-blind peer review.

Compliance with Ethics: This article involves a review of literature and does not report on new clinical data, or any studies with human or animal subjects performed by either of the authors.

Authorship: The named authors meet the International Committee of Medical Journal Editors (ICMJE) criteria for authorship of this manuscript, take responsibility for the integrity of the work as a whole, and have given final approval for the version to be published.

Access: This article is freely accessible at touchCARDIO.com (c) Touch Medical Media 2020.

Received: 2 March 2020

Accepted: 25 March 2020

Published Online: 19 June 2020

Citation: Heart International. 2020;14(1):20-3

Corresponding Author: Marco Alings,

PO Box 90158, 4800 RK Breda,

The Netherlands. E: MAlings@amphia.nL

Support: No funding was received for the publication of this article.
The factor Xa (FXa) inhibitors apixaban, rivaroxaban and edoxaban, together with the thrombin inhibitor dabigatran, are collectively referred to as non-vitamin $\mathrm{K}$ antagonist (or direct) oral anticoagulants (NOACS). FXa inhibitors are widely used for stroke prevention in patients with nonvalvular atrial fibrillation and for the treatment and prevention of deep vein thrombosis and pulmonary embolism. ${ }^{1-3}$ In patients with nonvalvular atrial fibrillation, NOACs have been reported to significantly reduce stroke or systemic embolic events compared with warfarin (relative risk [RR] 0.81, 95\% confidence interval [CI] 0.73-0.91; $p<0.0001$ ), with reduction in haemorrhagic stroke (RR 0.49 , 95\% $\mathrm{Cl} 0.38-0.64 ; \mathrm{p}<0.0001$ ) being the main driver of stroke reduction. ${ }^{4}$ In patients with venous thromboembolism, which includes deep vein thrombosis and pulmonary embolism, NOACs have comparable efficacy to vitamin $\mathrm{K}$ antagonists, with no significant difference in recurrence of venous thromboembolism (RR $0.88,95 \% \mathrm{Cl} 0.74-1.05 ; \mathrm{p}=0.46$ ), fatal pulmonary embolism (RR 1.02 , 95\% Cl 0.39-5.96; $p=0.71$ ) or all-cause mortality (RR 0.97, 95\% Cl 0.83-1.14; $p=0.50) .{ }^{5}$

Bleeding is a feared complication of anticoagulant therapy. Warfarin-related bleeding is responsible for one-third of all hospitalisations for adverse drug events and the mortality rate of patients hospitalised with vitamin Kantagonist-related bleeding was $7.6 \%$, with a 90 -day mortality of $14.1 \% .{ }^{6,7}$ Both in patients with nonvalvular atrial fibrillation and those with venous thromboembolism, NOACs are associated with a lower risk of major bleeding compared with warfarin. In particular, NOACs were associated with a lower risk of intracranial or life-threatening bleeding. ${ }^{4,-11}$

Despite their demonstrated safety, the lack of a specific antidote may have limited the implementation of FXa inhibitors in clinical practice. However, andexanet alfa, the specific antidote for the reversal of FXa inhibitor activity, has recently been developed..$^{12}$ The focus of this review will be on the position of andexanet alfa within the management of FXa inhibitor-associated bleeding.

\section{Andexanet alfa as antidote for factor Xa inhibitors}

Direct FXa inhibitors share a relative short elimination half-life of approximately 12 hours. Rivaroxaban and edoxaban are administered once-daily and apixaban is administered twice-daily. Renal clearance is $35 \%, 50 \%$ and $27 \%$, respectively. ${ }^{13}$

Andexanet alfa is designed to reverse the direct and indirect anticoagulant activity of FXa inhibitors. ${ }^{14}$ Andexanet alfa is a modified, recombinant, inactive form of human FXa, with the ability to bind and sequester FXa-inhibitor molecules, thereby reducing its activity and restoring the amount of unbound endogenous FXa. However, andexanet alfa binds to tissue factor pathway inhibitors as well, which may lead to increased thrombin generation.

Andexanet alfa therapy is delivered by an intravenous (IV) bolus over a duration of 15-30 minutes, followed by an infusion lasting 2 hours. Prior to the initiation of andexanet 
Table 1: Low-dose andexanet alfa

\begin{tabular}{|l|l|l|}
\hline Rivaroxaban dose & Apixaban dose & Timing from last dose \\
\hline$\leq 10 \mathrm{mg}$ OD & $\leq 5 \mathrm{mg}$ BID & Any time \\
\hline$>10 \mathrm{mg}$ OD & $>5 \mathrm{mg}$ BID & $\geq 8$ hours \\
\hline Unknown & Unknown & $\geq 8$ hours \\
\hline
\end{tabular}

$B I D=$ twice-daily; $O D=$ once-daily.
Table 2: High-dose andexanet alfa

\begin{tabular}{|l|l|l|}
\hline Rivaroxaban dose & Apixaban dose & Timing from last dose \\
\hline$>10 \mathrm{mg}$ OD & $>5 \mathrm{mg}$ BID & $<8$ hours \\
\hline Unknown & Unknown & $<8$ hours \\
\hline
\end{tabular}

$B I D=$ twice-daily; $O D=$ once-daily.

Figure 1: Algorithm for the management of uncontrolled or life-threatening bleeding and appropriate use of andexanet alfa

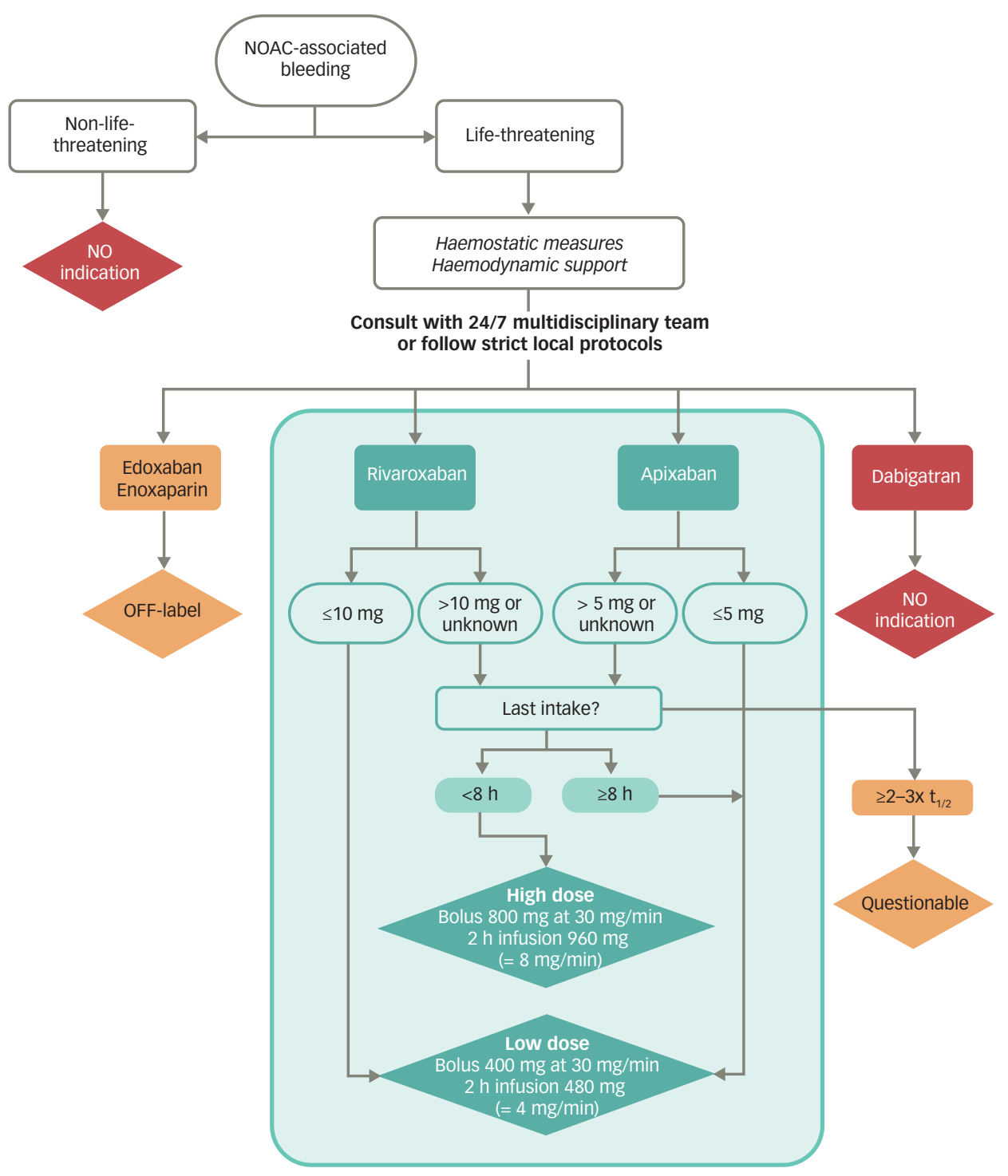

Following ISTH and BARC definitions, life-threatening bleeding encompasses probable fatal bleeding (BARC 5a), bleeding in a critical organ such an intracranial bleeding (BARC 3C), cardiac tamponade, bleeding requiring surgical intervention for control and bleeding requiring IV vasoactive agents (BARC $3 \mathrm{~b}){ }^{28}$

$B A R C=$ Bleeding Academic Research Consortium; $h=$ hours; ISTH = International Society on Thrombosis and Haemostasis; $I V=$ intravenous; min = minute;

NOAC $=$ non-vitamin $\mathrm{K}$ antagonist oral anticoagulants.

alpha, the dose is adjusted according to the last dose of FXa inhibitor, and timing from the last intake of FXa inhibitor (Table 1 and Table 2; Figure 1). ${ }^{15}$ Low dose consists of an IV bolus of $400 \mathrm{mg}$ at $30 \mathrm{mg} / \mathrm{min}$, followed by an infusion of $480 \mathrm{mg}$ at $4 \mathrm{mg} / \mathrm{min}$. High dose consists of a double bolus of $800 \mathrm{mg}$ followed by an infusion of $960 \mathrm{mg}$ at $8 \mathrm{mg} / \mathrm{min}$.

The dosing recommendations in the andexanet alfa product characteristics do not contain a maximum time from last dose of rivaroxaban or apixaban after which treatment with andexanet alfa could be considered futile. From a pharmacodynamic and clinical point of view, however, the clinical usefulness of treatment with andexanet alfa $>2-3$ times the half-lives after last dose of rivaroxaban ( $t_{1 / 2} 5-13$ hours) or apixaban ( $t_{1 / 2} 13$ hours), seems questionable.

In healthy volunteers, 50-75 years of age and treated with apixaban or rivaroxaban, bolus administration of andexanet alfa reduced anti-FXa activity and restored thrombin generation within 2-5 minutes. ${ }^{16}$ 
When andexanet alfa was administered as bolus plus infusion, consistent with its half-life of $\sim 1$ hour, the effects were sustained and then waned after 2 hours. In a subgroup of participants, transient increases in levels of D-dimer and prothrombin fragments 1 and 2 resolved within 24-72 hours without thrombotic events. ${ }^{16}$

ANNEXA-4 was a single-group cohort study that evaluated the clinical utility of andexanet alfa in patients who experienced acute major bleeding within 18 hours after administration of apixaban (54\%), rivaroxaban (40\%) or enoxaparin (6\%). ${ }^{17}$ Sixty-four percent of patients suffered from intracranial bleeding and $26 \%$ from gastrointestinal bleeding. Patients were treated with a bolus of andexanet alfa, followed by a 2-hour infusion. Treatment with andexanet alfa resulted in a $92 \%$ reduction of anti-FXa activity. In $85 \%$ of patients with gastrointestinal bleeding and in $80 \%$ of patients with intracranial bleeding, 'excellent' or 'good' haemostatic efficacy was observed at 12 hours, as adjudicated according to prespecified criteria. Within 30 days, $14 \%$ of the patients had died. Thrombotic events occurred in $10 \%$ of patients, none of whom had yet restarted oral anticoagulation. ${ }^{17}$

Based on the results of the ANNEXA-4 study, the use of andexanet alfa has been conditionally approved by both the US Food and Drug Administration and the European Medicines Agency for adult patients treated with a direct FXa inhibitor (apixaban or rivaroxaban) when reversal of anticoagulation is needed due to life-threatening or uncontrolled bleeding. ${ }^{15,18}$ However, the risk-benefit ratio of andexanet alfa in comparison to usual care remains to be determined. As this is a condition for accelerated approval in the USA, a randomised clinical trial is currently ongoing to determine the efficacy and safety of andexanet alfa compared to usual care in patients presenting with acute intracranial haemorrhage within 12 hours of symptom onset and within 15 hours of taking an oral FXa inhibitor, with an estimated completion date in 2023. ${ }^{19}$

Use of andexanet alfa in the setting of mild or non-life-threatening bleeding, for patients undergoing urgent or immediate high-risk surgery, or use of andexanet alfa for other FXa inhibitors than apixaban or rivaroxaban, is off-label and is the subject of ongoing studies. In preclinical studies, andexanet alfa effectively reversed the anticoagulant effect of other FXa inhibitors. ${ }^{20,21}$ Clinical data of andexanet alfa in patients requiring urgent surgery and receiving apixaban, rivaroxaban, edoxaban or enoxaparin within 15 hours prior to the start of surgery, are currently being evaluated in a prospective open-label study.22

\section{Practical considerations}

With approval of idarucizumab in 2015 and andexanet alfa in 2019, effective direct anticoagulant reversal agents are now available for patients taking dabigatran, apixaban or rivaroxaban. ${ }^{23}$ With the widespread availability of these effective anticoagulant reversal agents, the correct use of these agents in real-world care needs special attention. The decision to use reversal agents for NOACs is most likely to be made in the acute critical care setting, in which prompt decisions are required. Inappropriate use unnecessarily exposes patients to (thrombotic) risks, and increases healthcare costs.

The clinical settings in which idarucizumab is used in real-life care, in all probability, are a good reflection of the clinical situations in which andexanet alfa will be used as well. The appropriate usage of idarucizumab in daily clinical practice was evaluated in a retrospective observational study by van der Wall et al. ${ }^{24}$ Between 2016 and 2018, 88 patients were treated with idarucizumab in 12 Dutch hospitals. The number of administrations of idarucizumab varied from 1-14 during the 2-year study period. Inappropriate use of idarucizumab occurred in 25 of the patients (28\%): in 14 patients for an intervention that could have been delayed for at least 8 hours, and in 11 patients for bleeding complications that were not considered uncontrollable. Three patients (5.7\%) had non-detectable dabigatran plasma levels, in two of these patients the last drug intake was $>72$ hours previously and one patient used rivaroxaban.

These data demonstrate the importance of introducing a local treatment protocol for the appropriate use of direct anticoagulant reversal agents, correctly determining the severity of the bleeding event or the urgency and bleeding risk of surgical procedures, and the importance of assessing the type, dosage and time of the last intake of the NOAC.

Routine coagulation tests (prothrombin time, activated partial thromboplastin time and international normalised ratio) do not provide an accurate assessment of the anticoagulant effects of FXa inhibitors. However, in case of serious bleeding, coagulation tests may help the clinician to support haemostasis. Anticoagulant effects can be measured via specific coagulation assays developed for the quantification of NOAC plasma levels. Most routine coagulometers are capable of measuring NOAC plasma levels within $\leq 30$ minutes. ${ }^{25}$ Healthcare institutions are recommended to consider $24 / 7$ availability of these tests for emergency situations. Absence of anti-Xa activity with chromogenic assays excludes clinically relevant drug levels. Protamine sulphate and vitamin $\mathrm{K}$ are not expected to affect the anticoagulant activity of rivaroxaban or apixaban. There is limited experience with tranexamic acid or prothrombin complex concentrates (PCCs).

In a meta-analysis of 10 single-arm studies in 340 patients and a retrospective observational study of 31 patients who received PCC for FXa inhibitor-related major bleeding, effective haemostasis was reported in $69 \%$ and $81 \%$, respectively. 26,27 Based on non-placebo-controlled reports, however, it is difficult to determine whether PCC, in addition to cessation of direct oral FXa inhibitor, is more effective than cessation of direct oral FXa inhibitor alone. To the best of our knowledge, there are no randomised trials comparing PCC to andexanet alfa. The decision to administer andexanet alfa should be made in consultation with a multidisciplinary team who should be available 24/7 and consist of a coagulation expert, a cardiologist specialising in anticoagulation, a vascular internal medicine physician and a clinical chemist.

Local protocols should be available describing the treatment of patients using FXa inhibitors with uncontrolled or life-threatening bleeding, including haemostatic measures, haemodynamic support and the use of andexanet alfa. According to the Committee for Medicinal Products for Human Use, the dose of andexanet alfa is first determined by the last dose of rivaroxaban or apixaban before initiation of andexanet alfa. By default, patients treated with apixaban for stroke prevention in atrial fibrillation will always use an apixaban dose $\leq 5 \mathrm{mg}$. Hence, these patients will always receive the low dose of andexanet alfa. Likewise, patients using rivaroxaban $\leq 10 \mathrm{mg}$ will always receive the low dose of andexanet alfa. In patients using higher doses of apixaban or rivaroxaban, the timing from last intake before initiation of andexanet alfa determines the dose of andexanet alfa (Figure 1).$^{28} \square$ 
1. Kirchhof P, Benussi S, Kotecha D, et al. 2016 ESC Guidelines for with EACTS Europace 2016:18:1609-78.

2. Konstantinides SV, Meyer G et al. 2019 ESC Guidelines for management of acute pulmonary embolism developed in collaboration with the European Respiratory Society (ERS): The Task Force for the diagnosis and management of acute pulmonary embolism of the European Society of Cardiology (ESC). Eur Heart J. 2019; doi:10.1183/13993003.01647-2019.

3. Mazzolai L, Aboyans V, Ageno W, et al. Diagnosis and management of acute deep vein thrombosis: a joint consensus document from the European Society of Cardiology working groups of aorta and peripheral vascular diseases and pulmonary circulation and right ventricular function. Eur Heart J. 2018;39:4208-18.

4. Ruff CT, Giugliano RP, Braunwald E, et al. Comparison of the efficacy and safety of new oral anticoagulants with warfarin in patients with atrial fibrillation. a meta-analysis of randomised trials. Lancet. 2014;383:955-62

5. van der Hulle T, Kooiman J, den Exter PL, et al. Effectiveness and safety of novel oral anticoagulants as compared with vitamin $\mathrm{K}$ antagonists in the treatment of acute symptomatic venous thromboembolism: a systematic review and meta-analysis. Thromb Haemost. 2014:12:320-8.

6. Budnitz DS, Lovegrove MC, Shehab N, Richards CL. Emergency hospitalizations for adverse drug events in older Americans. N Engl J Med. 2011; 365:2002-12

7. Halbritter K, Beyer-Westendorf J, Nowotny J, et al. Hospitalization for vitamin-K-antagonist-related bleeding: treatment patterns and outcome. J Thromb Haemost. 2013;11:651-9.

8. Connolly SJ, Ezekowitz MD, Yusuf S, et al. Dabigatran versus warfarin in patients with atrial fibrillation. $N$ Eng/ I Med. 2009:361:1139-51.

9. Granger $\mathrm{CB}$, Alexander $\mathrm{JH}$, MCMurray JJ, et al. Apixaban versus warfarin in patients with atrial fibrillation. $N$ Engl J Med. 2011:365:981-92.
10. Patel MR, Mahaffey KW, Garg J, et al. Rivaroxaban versus warfarin in nonvalvular atrial fibrillation N Eng/ $J$ Med. 2011;365:883-91.

11. Giugliano RP, Ruff $\mathrm{CT}$, Braunwald $\mathrm{E}$, et al. Edoxaban versus warfarin in patients with atrial fibrillation. N Eng/ / Med. 2013:369:2093-104.

12. Ghadimi K, Dombrowski KE, Levy JH, et al. Andexanet alfa for the reversal of factor $X a$ inhibitor related anticoagulation Expert Rev Hematol. 2016;9:115-22.

13. Heidbuchel H, Verhamme P, Alings M, et al. European Hear Rhythm Association Practical Guide on the use of new oral anticoagulants in patients with non-valvular atrial fibrillation. Europace. 2013;15:625-51.

14. Lu G, DeGuzman FR, Hollenbach SJ, et al. A specific antidote for reversal of anticoagulation by direct and indirect inhibitors of coagulation factor Xa. Nat Med. 2013;19:446-51.

15. European Medicines Agency. Ondexxya ${ }^{\oplus}$, INN-andexanet alfa, summary of product characteristics. 2019. Available at: www.ema.europa.eu/en/documents/product-information/ ondexxya-epar-product-information_en.pdf (accessed 9 April 2020)

16. Siegal DM, Curnutte JT, Connolly SJ, et al. Andexanet alfa for the reversal of factor Xa inhibitor activity. N Engl I Med. 2015;373:2413-24

17. Connolly SJ, Crowther M, Eikelboom JW, et al. Full study report of andexanet alfa for bleeding associated with factor Xa Inhibitors. N Eng/J Med. 2019;380:1326-35.

18. Portola Pharmaceuticals. US FDA approves Portola Pharmaceuticals' Andexxa® ${ }^{\circledR}$, first and only antidote for the reversal of factor Xa inhibitors. 2018. Available at: www.globenewswire.com/news-release/2018/05/04/ 1496534/0/en/U-S-FDA-Approves-Portola-PharmaceuticalsAndexxa-First-and-Only-Antidote-for-the-Reversal-of-Factor-X Inhibitors.html (accessed 8 April 2020)

19. ClinicalTrials. gov. Trial of andexanet in ICH patients receiving an oral FXa inhibitor. 2019. Available from: www.clinicaltrials.gov/ ct2/show/NCT03661528 (accessed 29 March 2020).
20. Lu G, Pine P, Leeds JM, et al. Andexanet alfa effectively reverses edoxaban anticoagulation effects and associated eveding in a rabbit acute hemorrhage model PLOS One. 2018;13:e0195122.

21. Fareed 1 , Hoppenstaedt DA, et al. Factor Xa inhibitor antidote, andexanet alfa differentially reverses the effects of apixaban, betrixaban, edoxaban and rivaroxaban. Circulation. 2019;140:A13765

22. ClinicalTrials.gov. Trial of andexanet in patients receiving an oral FXa inhibitor who require urgent surgery (Annexa-S). 2020 Available from: www.clinicaltrials.gov/ct2/show/NCT04233073 (accessed 29 March 2020).

23. Schiele F, van Ryn J, Canada K, et al. A specific antidote for dabigatran: functional and structu characterization. Blood. 2013;121:3554-62.

24. van der Wall SJ, van Rein N, van den Bemt B et al. Performance of idarucizumab as antidote of dabigatran in daily clinical practice. Europace. 2019:21:414-20.

25. Steffel $\perp$, Verhamme P, Potpara TS, et al. The 2018 European Heart Rhythm Association Practical Guide on the use of non-vitamin $\mathrm{K}$ antagonist oral anticoagulants in patients with non-vitamin K antagonist oral anticoagulants in pabrillation. Eur Heart J. 2018:39:1330-93.

26. Piran S, Khatib R, Schulman S, et al. Management of direct factor Xa inhibitor-related major bleeding with prothrombin complex concentrate: a meta-analysis. Blood Adv. 2019;3:158-67.

27. Smith MN, Deloney L, Carter C, et al. Safety, efficacy, and cost of four-factor prothrombin complex concentrate (4F-PCC) in patients with factor Xa inhibitor-related bleeding: a retrospective study. J Thromb Thrombolysis. 2019;48:250-5.

28. Mehran R, Rao SV, Bhatt DL, et al. Standardized bleeding definitions for cardiovascular clinical trials: A consensus report from the Bleeding Academic Research Consortium. Circulation. 2011:123:2736-47. 